\title{
Antimicrobial Resistance in Microorganisms
}

\author{
Mohammad Reza Arabestani ${ }^{1,2}$; Manoochehr Karami ${ }^{3}$; Mohammad Yousef Alikhani ${ }^{1,2,}$ \\ ${ }_{1}^{1}$ Department of Microbiology, Faculty of Medicine, Hamadan University of Medical Sciences, Hamadan, IR Iran \\ ${ }^{2}$ Brucellosis Research Center, Hamadan University of Medical Sciences, Hamadan, IR Iran \\ 3 Department of Biostatistics and Epidemiology, School of Public Health, Hamadan University of Medical Sciences, Hamadan, IR Iran \\ ${ }^{*}$ Corresponding author: Mohammad Yousef Alikhani, Brucellosis Research Center, Hamadan University of Medical Sciences, Hamadan, IR Iran. Tel: +98-8118380755, Fax: +98- \\ 8118380130, E-mail: alikhani@umsha.ac.ir
}

Received: May 1, 2014; Accepted: May 1, 2014

Keywords: Drug Resistance, Microbial; World Health Organization; Infection Control

The World Health Organization has warned that presently there is a real threat posed by antimicrobial resistant (AMR) organisms. Antimicrobial resistance is resistance of a microorganism to an antimicrobial medicine to which it was originally sensitive (1). Antibiotic resistance has been detected in microbes that cause common and serious diseases, such as urinary tract infections and pneumonia in all regions of the world. This means that when we are most vulnerable and in need of these drugs, there is a chance that they may not work as currently antimicrobial resistance threatens the effective avoidance and treatment of an ever-increasing range of infections caused by bacteria, parasites, viruses and fungi. Resistant organisms are able to withstand attacks by antimicrobial medicines, such as antibiotics, so that standard treatments become ineffective and infections persist, increasing risk of spread to others. The evolution of resistant strains is a natural phenomenon that occurs when microorganisms are exposed to antimicrobial drugs, and resistant traits can be exchanged between certain types of bacteria (2). The misuse and overuse of antimicrobial medicines accelerates this natural phenomenon. Poor infection control practices encourage the spread of AMR. In general, bacterial resistance to antimicrobial agents is classified into multidrug-resistant (MDR), extensively drug-resistant (XDR) and pandrug-resistant (PDR); MDR has been defined as acquired non-susceptibility to at least one agent in three or more antimicrobial categories, XDR means bacterial isolates remain susceptible to only one or two categories and PDR has been defined as non-susceptibility to all agents in all antimicrobial categories (3).

When bacteria become resistant to an antibiotic, that medicine becomes less effective. Medical treatment of patients infected with these drug-resistant organisms can become more complicated, leading to longer hospital stays, increased health care costs and in extreme cases, untreatable infections. Without effective antimicrobials for care and prevention of infections, the success of treatments such as organ transplantation, cancer chemotherapy and major surgery would be compromised. Generally speaking, the growth of global trade and travel allows resistant microorganisms to spread rapidly to distant countries and continents through humans and food (4).

\section{Acknowledgements}

There is no acknowledgement.

\section{Authors' Contribution}

All authors contribute in this research the same.

\section{Financial Disclosure}

There is no conflict of interest.

\section{References}

1. Antimicrobial resistance: global report on surveillance 2014. 2014 Available from: http://www.who.int/drugresistance/documents/ surveillancereport/en.

2. Falagas ME, Koletsi PK, Bliziotis IA. The diversity of definitions of multidrug-resistant (MDR) and pandrug-resistant (PDR) Acinetobacter baumannii and Pseudomonas aeruginosa. J Med Microbiol. 2006;55(Pt 12):1619-29.

3. Kuo LC, Teng LJ, Yu CJ, Ho SW, Hsueh PR. Dissemination of a clone of unusual phenotype of pandrug-resistant Acinetobacter baumannii at a university hospital in Taiwan. J Clin Microbiol. 2004;42(4):1759-63.

4. Park YK, Peck KR, Cheong HS, Chung DR, Song JH, Ko KS. Extreme drug resistance in Acinetobacter baumannii infections in intensive care units, South Korea. Emerg Infect Dis. 2009;15(8):1325-7. 